\title{
Kyste osseux anévrysmal mandibulaire : un diagnostic qui reste difficile.
}

\author{
Derruau S1, Fleury C², Cervellera C1, Touche D³, Laurence S1, Lefèvre B1, Mauprivez C1 \\ 1. Pôle Odontologie, Hôpital Maison Blanche - $\mathrm{CHU}$ Reims \\ 2. Laboratoire de Biopathologie, Hôpital Robert Debré, CHU Reims \\ 3. Service de Radiologie, Institut Jean Godinot, Reims
}

Le kyste osseux anévrysmal (KOA) est une lésion osseuse rare des os longs et du rachis, d'étiologie indéterminée, considérée comme bénigne, dont la localisation faciale est exceptionnelle (2\%). II survient le plus souvent chez des patientes jeunes (10-20ans) avec un ratio localisation mandibulaire / maxillaire de $3: 1$, représentant $1.5 \%$ des kystes non odontogéniques (Robert $A, 2012)$. Les localisations mandibulaires sont le corps, l'angle et le ramus. La présentation clinique du KOA est variable, d'une petite lésion asymptomatique de découverte fortuite à une lésion ostéolytique douloureuse volumineuse, pouvant rompre les corticales et responsable d'une malocclusion.

Le but de ce travail a été de rapporter les données cliniques, radiologiques et histologiques d'une patiente ayant présenté un kyste anévrysmal mandibulaire.

Le cas d'une patiente âgée de 60ans est rapporté, sans antécédent, adressée pour découverte fortuite d'une image radioclaire mandibulaire. L'orthopantomogramme objectivait une lésion ostéolytique uniloculaire au niveau du corpus mandibulaire gauche étendue de la face mésiale de la première prémolaire à la face distale de la deuxième molaire sans rapport avec les apex dentaires. Un examen volumétrique (Cone Beam Computed Tomography, $\mathrm{CBCT}$ ) montrait une image à contour net avec un respect des corticales vestibulaire et linguale. Le nerf alvéolaire inférieur gauche semblait avoir un trajet au sein de la lésion et le canal mandibulaire paraissait être en partie dévié dans sa partie postérieure. L'imagerie par résonnance magnétique (IRM) mettait en évidence une lésion osseuse en hypersignal T1, signal voisin de l'os spongieux mais inférieur à celui de la graisse, et un hyposignal T2 équivalent au signal de l'air. En T2, le pédicule vasculo-nerveux alvéolo-inférieur était parfaitement individualisé du reste de la lésion sans anomalie le long de son trajet intra-osseux. Une exploration chirurgicale en vue d'une exérèse complète est réalisée sous anesthésie générale. En per-opératoire, la lésion macroscopique apparaissait comme une cavité osseuse déshabitée à contenu hétérogène, avec un tissu fibreux périnerveux et la présence de quelques lacs vasculaires. L'examen microscopique objectivait des fragments de tissu osseux lamellaire individualisant des espaces contenant un tissu fibro-adipeux et congestif avec par endroits un tissu de granulation hypervascularisé avec des capillaires à paroi épaissie fibreuse associés à une paroi fibrohyaline et des suffusions hémorragiques. La synthèse des données cliniques, radiologiques et histologiques a permis d'établir le diagnostic de kyste osseux anévrysmal. Un suivi clinique et radiologique a été instauré.

Les lésions ostéolytiques pures de la mandibule non odontogènes posent d'importants problèmes de diagnostic et de prise en charge thérapeutique. Si l'examen histopathologique permet d'écarter avec certitude un granulome à cellules géantes, un lipome ou un angiome, l'absence de nombreux lacs ou sinusoïdes remplis de sang rend l'histologie d'interprétation plus difficile pour la distinction entre le KAO et le kyste osseux solitaire (Triantafillidou, 2012). En définitive, le diagnostic de KAO reste de diagnostic difficile, le plus souvent d'exclusion reposant sur la confrontation de l'ensemble des données cliniques, radiologiques et histologiques.

stephane.derruau@gmail.com

(C) The authors, published by EDP Sciences. This is an Open Access article distributed under the terms of the Creative Commons Attribution License 4.0 (http://creativecommons.org/licenses/by/4.0/). 